\title{
Consecuencias semánticas de transformaciones gráficas en un poema de José Emilio Pacheco
}

\section{Semantic Consequences of Graphic Transformations in a Poem by Jose Emilio Pacheco}

\author{
Raúl Dorra \\ Benemérita Universidad Autónoma de Puebla, México
}

\begin{abstract}
Resumen: Con un método cercano a la estilística, este artículo desarrolla un análisis comparativo de dos versiones de un poema de José Emilio Pacheco. Tales versiones presentan la particularidad de que la segunda repite las mismas palabras de la primera, pero modifica la organización de los versos, con lo cual se modifica la imagen visual del poema y también la entonación de la voz en la lectura. El análisis de ambas versiones propone mostrar cómo una transformación en el nivel gráfico produce transformaciones en el nivel semántico. De este análisis se deriva una reflexión general acerca de la escritura.

Palabras clave: enunciación, escritura, voz, mirada, sujeto.
\end{abstract}

Abstract: With a method similar to stylistics, this article performs a comparative analysis of two versions of a poem by José Emilio Pacheco. These versions have once peculiarity, the second one repeats the same words of the first one but modifies the organization of the verses, thereby modifying the visual image of the poem as well as the intonation of the voice when reading it. The analysis of both versions aims at showing how a transformation in the graphic level causes transformations in the semantic level. This analysis leads to a general reflection about writing.

Keywords: Enunciation, Writing, Voice, Look, Subject.

Recibido: 31 diciembre de 2016

Aceptado: 17 de marzo de 2017 


\section{A modo de introducción}

U na notable característica del oficio de escritor de José Emilio Pacheco es su tal vez irreprimible tendencia a modificar los textos antes de entregarlos para una nueva edición. Las modificaciones pueden ser más o menos cuantiosas y afectar a la vez a varios niveles de la lengua, o pueden ser menores y reducirse, en el caso de sus poemas, a la organización gráfica del enunciado, lo cual, desde luego, no deja de tener consecuencias significantes. Es como si Pacheco continuamente desoyera la conminación que Juan Ramón Jiménez, en el minipoema que abre su libro Piedra y cielo, dirige a los poetas afanados en la corrección de sus versos: “iNo le toques ya más, / que así es la rosa!” (Jiménez, 2008: 47).

No resulta difícil asociar esta tendencia a modificar los textos, esta movilidad de su escritura, a ese agudo y permanente sentimiento de las transformaciones que opera el tiempo en la materia y por lo tanto, o sobre todo, también en el espíritu. Sus libros de poemas lo anuncian ya desde su título y el libro en que recoge su obra poética (libro que el poeta mantuvo siempre en transformación) se llama, con toda coherencia: Tarde o temprano. En un breve poema, Pacheco pone en boca de José Luis Cuevas estas palabras que resumen, más que la mirada del pintor, su propia mirada de poeta: "Mi desolado tema / es ver qué hace la vida / con la materia humana" (Pacheco, 2009: 123). ¿Cómo aceptar, entonces, la propuesta de Juan Ramón Jiménez según la cual el poema, como la rosa, no es un hacer y un deshacer sino un hecho definitivo? ¿Cómo, si las mismas palabras que componen esos dos versos definitivos del poeta español tienden, por una especie de gravedad, a cambiar de lugar y a modificar su ritmo y su sentido?

Yo conservo en mi memoria esos versos de Juan Ramón Jiménez siempre transformados. Cuando los cito de memoria, o los oigo citar, tienden a sufrir algunos desplazamientos. Por ejemplo: 
"Ya no la toques más / que así es la rosa". Aquí por ejemplo el desplazamiento del adverbio desde la penúltima a la primera posición silábica ha alterado el ritmo del verso, y la transformación del pronombre (la por le) ha alterado su sentido. O, como he oído citar a otros: "Que no le toques más: / así es la rosa". Ese emblemático par de versos del poeta de Moguer ha generado, a su pesar y por su pesar, éstas y otras variantes con las que la memoria, al retenerlos, se entretiene. Así pues, si las cosas ocurren entre la realidad y el deseo tendríamos que decir que Juan Ramón Jiménez es un poeta del deseo y José Emilio Pacheco es un poeta de la realidad aunque se trate de una definición exagerada en ambos casos.

\section{Estudio comparativo. El proceso de enunciación}

Ahora bien, después de esta suerte de introducción, me interesa decir que en este trabajo volveré sobre un estudio comparativo de dos versiones de un mismo poema de Pacheco: "Digamos que Amsterdam 1943". ${ }^{1}$ Dicho estudio está en un libro publicado en el milenio del que nos hemos desprendido hace ya dieciséis años pero que nunca, al parecer, acabará de irse. El libro al que me refiero está dedicado al examen de Morirás lejos (Dorra, 1986), un texto que sólo por comodidad o impotencia hemos venido llamando novela, clasificación que tiende a no dejar ver que está construido, como lo ha observado Gustavo Lespada (2002) siguiendo un principio de indeterminancia cuya estructura consiste en una continua desestructuración. En esa obra de Pacheco, más de una vez, la página aparece como espacio en el que se distribuyen las grafías y, por lo tanto, se muestra tratada según un principio que asociamos casi

${ }^{1}$ La primera versión está tomada de la primera edición de No me preguntes cómo pasa el tiempo, hecha por Joaquín Mortiz en 1969, y la segunda de la primera edición de Tarde o temprano, reunión de la obra poética de Pacheco, hecha por el Fondo de Cultura Económica en 1980. 
naturalmente con el poema. Por otra parte, si Morirás lejos es una obra caracterizada por la inestabilidad en todos sus niveles, como obedeciendo a esa gravedad, la segunda edición fue una minuciosa, y en muchos casos invisible, transformación de la primera. Yo me he detenido en el cotejo de esas dos ediciones hechas por Mortiz respectivamente en los ańos de 1967 y 1977, y encontré que la segunda edición "presenta en términos generales un desplazamiento en dos direcciones convergentes: hacia una simplificación del enunciado y hacia una politización del texto" (Dorra, 1986: 215). Como se sabe, Morirás lejos es una obra que se mueve entre lo testimonial y lo literario y avanza en ambas direcciones tratando de mostrar que ese avance es imposible y al mismo tiempo imprescindible. Tal vez con alguna conciencia de culpa, la mano que ha corregido la primera versión, ha buscado una relativa atenuación del aspecto "literario" en proporción directa a la acentuación del aspecto "testimonial". Pero para lo que ahora nos interesa diremos aquí que las modificaciones hechas a la segunda edición son también obra del tiempo.

Veamos las dos versiones:

¿Diremos que estamos ante otro acto verbal? Yo sin dudas diría que sí, que necesariamente esa nueva disposición gráfica del poema tiene que tener consecuencias en la significación verbal. De tal modo, es siempre posible reunir en un mismo proceso significante el aporte de signos de naturaleza gráfica o, en general, de orden visual. Eso me enseñó este poema de Pacheco al mostrarme dos versiones visuales que eran también, o esencialmente, dos actos verbales situados no en el habla sino en la escritura. Aquí lo que habla es la escritura. Haciendo este tipo de reflexiones en aquel primer cotejo consideraba yo que la poesía, en términos estrictos, nos sitúa ante lo hablado en estado puro, esto es, ante una materialidad constituida como un proceso fundante. Y que si esto es así, debemos observar que en esa materialidad se inscriben, además de 
los signos verbales propiamente dichos, otros signos que no tienen su origen en la lengua sino en el discurso escrito e incluso en las diferentes modalidades que dan forma a este discurso. Textualmente cito: "La palabra escrita es también trazo, extensión, sonoridad, y esas propiedades se articulan en lo concreto del discurso para incorporarse al proceso de su significación" (Dorra, 1986: 264). Saussure, que pensó que la escritura no era de interés para el lingüista, habló del significante como una imagen acústica. La fórmula "imagen acústica" parece encerrar una contradicción pues la imagen, al menos en principio, tiene una naturaleza visual. Tal vez recurrió a esa fórmula porque, como él mismo reconoce, aunque la escritura queda fuera del campo de la lingüística, esta ciencia no podría haberse constituido sin el auxilio de la escritura. La imagen acústica está pues de algún modo asociada -contaminada, diría Saussure- por una imagen visual. La poesía, sobre todo la poesía moderna, no sólo hace evidente esa relación sino que obliga a considerar que el significante tiene un aspecto visual. $\mathrm{Y}$ es ese aspecto visual el que, paradójicamente, nos permite idear una forma -o una imagen- acústica.

\subsection{Enunciación verbal y enunciación visual}

Como dije, en estos últimos años me he dedicado a observar en los textos escritos, sobre todo en los poéticos, cómo la escritura construye una voz que es el resultado de una relación de lo visible con lo audible y cómo cada una de estas dimensiones se relacionan con una negatividad que la sostiene: lo visible es tal en la medida en que se sostiene sobre lo invisible del mismo modo que lo audible es un aspecto emergente de lo inaudible. También la composición gráfica señala los espacios de silencio así como el fondo de invisibilidad sobre el cual se levanta. 


\section{Digamos QUe Amsterdam 1943}

EL AGUA vuelve al agua.

Qué inclemente

caer del agua sobre los canales

en la mañana inerme.

$\mathrm{Y}$ a lo lejos

un silbato de fábrica.

Entre sábanas, roto, envejeciendo, está el periódico:

la guerra continúa, la violencia incendia nuestros años.

Bajo tu cuerpo y en tu sueño duermes.

¿Qué será de nosotros? ¿Cuándo y dónde segará nuestro amor el tajo, el fuego?

Se escucha la respuesta:

están subiendo.

Me voy, no te despiertes:

los verdugos

han tocado a la puerta.
El agua vuelve al agua.

Qué inclemente

caer del agua sobre los canales en la mañana inerme. Y a lo lejos un silbato de fábrica.

Entre sábanas, roto, envejeciendo, está el periódico:

la guerra continúa, la violencia incendia nuestros años.

Bajo tu cuerpo y en tu sueño duermes. ¿Qué será de nosotros? ¿Cuándo y dónde segará nuestro amor el tajo, el fuego? Se escucha la respuesta: están subiendo. Me voy, no te despiertes: los verdugos han tocado a la puerta.

La voz, por su parte, es la presencia del sujeto en el momento de constituirse como tal: un quién que se hace cargo de la lengua y la convierte en habla. Ese quién no necesariamente tiene una identidad estable sino más bien móvil: es una entidad puntal y a la vez un proceso en el cual se incorporan otras voces: es un yo y un tú, un yo y un nosotros, un yo y un otro. Todo ese proceso se desarrolla en un devenir que es al mismo tiempo un continuo aquí y ahora.

Según estas consideraciones, el eje que guía mi trabajo de comparación y análisis es el recorrido que cumple el sujeto en tanto el quién, el qué y el cómo del habla. He copiado ambas versiones del poema y ahora trato de reducirlas a su pura configuración visual. Veámoslas: 


\section{Digamos QUe Amsterdam I943}
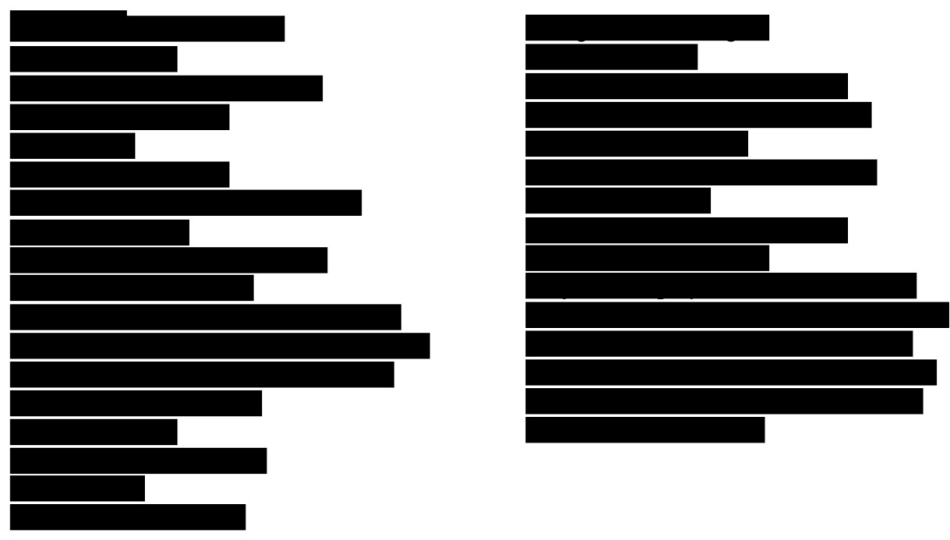

Hay que decir que esta réplica de lo que Noé Jitrik (2001) llamaría "la figura que reside en el poema" es puramente aproximativa y esencialmente equívoca desde el momento en que la duración de cada verso, así como su velocidad, es siempre móvil o inestable del mismo modo que la velocidad -o el tempo- del poema entero. La "figura" del poema, que estoy mostrando ahora, es una mecánica reproducción de las líneas según la extensión visual de cada verso pero esto no reproduce exactamente ni su cantidad silábica ni su duración temporal. Por ejemplo, si comparamos los dos primeros tetrasílabos ("Qué inclemente" con "y a lo lejos") pronto veremos que, dado que consta de una mayor cantidad de letras, el primero forma una línea de mayor extensión a pesar de tener la misma cantidad silábica. Y si observamos solamente el primer tetrasílabo veremos que éste tiene dos vocales tónicas y ofrece a la lectura la posibilidad de darle mayor intensidad a una o a otra: según ello, la extensión del período verbal resultará mayor o menor. Si consideramos -es decir, si quien se hace cargo de la lectura considera- que el acento principal debe caer sobre la primera vocal la duración 
será mayor porque habrá un detenimiento en el fluir prosódico mientras que si recae en la segunda vocal tónica el flujo será más rápido. La selección no es azarosa sino el resultado de una elección en el orden del sentido. Detenerse en el pronombre "Qué" supone enfatizar la perspectiva del sujeto, situarse en su espacio afectivo, mientras que enfatizar la tercera vocal de "inclemente" supone un debilitamiento de la perspectiva del sujeto, esto es: dirigir la mirada al paisaje, aun sin dejar de hacerse cargo de lo que este paisaje tiene de subjetivo. Por lo tanto, si quisiéramos representarnos la imagen de cada verso deberíamos tener al mismo tiempo en cuenta la extensión gráfica, la cantidad silábica, y la duración prosódica, aparte del tipo de velocidades de la modulación. Esto es imposible naturalmente, por lo menos si se trata del diseńo de una imagen única y fija. De ahí que el gráfico que aquí se muestra, si bien construye la imagen o el fantasma del verso, lo hace a costa de eliminar la vacilación o la indeterminación rítmica, que es esencial para el efecto poético. Al considerar, pues, cada verso, deberemos tener en cuenta tanto lo puntual como lo continuo, tanto las diferentes alturas como las diferentes velocidades de la curva melódica porque todo ello nos remite al acto enunciativo.

Revisemos ahora la primera versión incorporando la imagen visual. La imagen visual sugiere la línea del sonido y por esa vía reconduce al sentido. Siguiendo ese programa de lectura veremos que, desde el punto de vista del ritmo (aquello que interesa al oído en tanto es distribución de cláusulas acentuales y a la vista en tanto es administración de la materia gráfica), lo que domina es la combinación, característica de nuestra poesía clásica, de heptasílabos con endecasílabos, a los que se suman tetrasílabos fluctuantes, los cuales, incorporados al heptasílabo que lo precede o lo sucede, crean la posibilidad de construir nuevos endecasílabos. Digamos que el endecasílabo, y su armónica combinación con el heptasílabo para formar determinadas estrofas (liras, estancias, etc.), no 
es como en el caso de los metros usados por la poesía popular, un resultado de la pura melodía contenida en el habla sino que su historia está ligada a la escritura pues es una construcción tanto melódica como arquitectónica. Es también música para los ojos como lo comprendieron con agudeza Sor Juana o Quevedo, y sobre todo Góngora. En el caso de este poema, dicho ritmo crea la atmósfera propicia para un paisaje -exterior e interior-dominado por la caída del agua, el abandono, la inermidad, la resignada espera de lo ineluctable. Si observamos el movimiento que describen las palabras, encontraremos un ingrávido desplazamiento de lo exterior a lo interior y, hacia el final, una confrontación: alguien debe salir a la intemperie porque otro irrumpe en la interioridad.

Ahora bien, si nos preguntamos quién da cuenta de todo esto, desde dónde, en suma: quién habla, encontraremos que se trata de un sujeto cambiante, o más bien de un proceso en el que la subjetividad hablante es un desencadenamiento que, en términos generales, se mueve de lo extenso e ilocalizado a lo intenso y localizado. Yendo de la primera línea a la última, un rápido vistazo nos convencerá de que el que dice "El agua vuelve al agua" no es el mismo que dice: "los verdugos / han tocado a la puerta" aunque tampoco sea alguien por completo diferente. La expresión "El agua vuelve al agua" es una descripción del paisaje lluvioso pero antes de eso, y sobre todo después, es la evocación de una sentencia de origen bíblico: "El polvo vuelve al polvo". Esta sentencia evoca la fatalidad del continuo retorno a una dispersión primordial. Por lo tanto, quien habla en primer término lo hace para evocar aquella sentencia -que no le pertenece o más bien que le pertenece a él como a todos los hombres- y para que esa sentencia perdure, como un fondo, a lo largo del poema entero. El habla del poema comienza, se suelta -se desembraga, como se diría en semiótica- con estas palabras sentenciosas; pero la sentencia, por definición, es el resultado de un meditar, esto es del examen contemplativo de un estado 
de cosas. En consecuencia, en este poema la irrupción del habla la muestra como la desembocadura de un silencio que la precede, un silencio que no se interrumpe con el hablar sino que se mantiene sosteniéndola, que mantiene el hablar en lo inoído. Así, "El agua vuelve al agua" es una sentencia que dura proyectándose y a la vez instala aquello que precede a esa duración, un silencio primordial. Todavía podemos observar que en la primera versión la presencia del agua está resaltada por el uso de versalitas: "EL AGUA". El uso de las versalitas, hay que decir, es una convención de la editorial Joaquín Mortiz sin duda aceptada por el autor, convención que consiste en poner de relieve el íncipit de cada poema (en las ediciones que Era hizo de este libro ya no veremos las versalitas) lo cual no deja de incidir en la lectura. La presencia del agua necesariamente se optimiza aunque no haya sido precisamente ésa la intención al introducir este factor gráfico. Así, en este caso y por una suerte de azar, el agua está diciendo más, diciendo, también, otra cosa, remitiéndonos a una sentencia que se sitúa en un principio perdurable.

Algo semejante ocurre con el mirar. Si el que habla, el que habla en el poema, lo hace porque en verdad quiere decir, profundamente, otra cosa, el que mira el "inclemente" caer de la lluvia en realidad está igualmente tratando de ver otra cosa, algo que la lluvia sugiere y al mismo tiempo desplaza. Ver lo que no se deja ver, decir lo que no puede decirse es el intento del poema y acaso de toda forma del habla.

\subsection{La voz y la mirada}

Volviendo, pues, al proceso de la enunciación, tal vez quede más claro que éste incluye no sólo lo verbal sino también lo visual y que se trata de un desplazamiento de voces y miradas. Si nos fijamos bien, veremos que estamos situados ante distintos espacios de emplazamiento de la mirada y diversas propuestas de focalización, 
o quizá una sola pero en transformación, moviéndose de lo puramente abierto -el lugar sin localización desde donde se pronuncia la sentencia- a lo encerrado pero expuesto de una habitación, pasando por el espacio exterior que es a la vez un aqui donde cae la lluvia y un allá donde suena un silbato. Así, existen diversos horizontes del mirar y diversos objetos de la mirada. Dado, también, que el quién del habla se transforma (¿quién dice, por ejemplo, "la guerra continúa”?; ¡es la voz impersonal del periódico o la de un sujeto localizado?) entonces debemos suponer diversas flexiones de la voz. En realidad todo en el poema es desplazamiento ya sea en un sentido horizontal (lo que nos da un movimiento prospectivo tanto como retrospectivo), ya sea en un sentido vertical, esto es, en profundidad. Debajo del sustantivo "agua" leemos "polvo" y debajo aun "hombre" o "condición humana" mientras, por ejemplo, los adjetivos "inclemente" e "inerme", que describen y metaforizan respectivamente al "caer de lluvia" y a "la mañana” son en verdad una especie de hipálage, o sea un desplazamiento horizontal puesto que el efecto buscado se realiza cuando se asocia lo "inclemente" no con el "caer de lluvia" sino con la violencia -que es continua y dispersiva-, y lo "inerme" no con "la mañana" sino con "nuestro amor" el cual se encuentra construido sobre la indefensión. La escena de "nuestro amor" está hecha de transposiciones: la mirada envuelve a un cuerpo dormido, tratando de cubrirlo, y mantiene con él un diálogo inaudible que se instala entre la vigilia y el sueńo; el cuerpo nunca abandona aquel estado y es ese cubrirse con el sueño lo que devuelve, a quien mira, el sentimiento de la propia indefensión de su mirada. Este efecto de inversión se realiza también en las indicaciones temporales: el periódico que está "envejeciendo" informa de un hecho flagrantemente presente, mientras la pregunta por el "cuándo" y el "dónde", que de algún modo parece situar el final de "nuestro amor" en un todavía indeterminado alládespués, se resuelve en un abrupto aqui-ahora. En fin, entre otras 
transformaciones, el poema va de lo atópico a lo tópico, de lo contemplativo a lo activo, de la dispersión a la confrontación, de lo descriptivo -el paisaje lluvioso, la habitación donde se refugian los amantes- a lo narrativo: el paso de la reunión de los amantes a su dolorosa separación.

Tenemos, pues, un enunciado -lo que el poema literalmente dice- y por debajo un complejo trabajo de la enunciación que desplaza lo dicho y lo mirado. Todavía esta afirmación se refuerza si consideramos un elemento del poema, su título, cuyo estatuto es ambiguo porque, si bien no forma parte del cuerpo del poema, es el encargado de indicar la dirección de la lectura: "Digamos que Amsterdam 1943”. En este caso el título sugiere una circularidad así como una localización espacial y temporal que es esencialmente efímera. Ese título es una frase regida por un verbo que realiza una afirmación concesiva y al mismo tiempo diseminativa; una afirmación en la que el sujeto está buscando a otros con quien compartirla o en la que se desdobla en un monólogo. Débil si la leemos al comienzo, el recorrido del poema nos obliga a volver sobre ella y es entonces, al final del recorrido, cuando la frase impone todo su peso. La frase indica una fecha y un lugar, como si se tratara del periódico, y al mismo tiempo indica que esa fecha y ese lugar son también otras fechas y otros lugares. Entendemos de este modo también el envejecimiento del periódico, pues está dando siempre la misma noticia, aquí y allá, antes y después. Semejante al primer verso en cuanto a que la frase que sirve de título tiene una vigencia incesante, difiere de él en cuanto a que la fuerza del primer verso aparece en el momento mismo de su enunciación y la fuerza del título toma su plenitud al final. En cierto sentido, es como si el poema se iniciara en el primer verso y se cerrara -pues ahí vuelve a comenzar- con el título. Estas resonancias y transposiciones de lo audible así como estas dispersiones y convergencias 
de los desplazamientos visuales son lo que nos conduce a ese fondo de invisibilidad y de silencio al que el poema nunca deja de aludir.

Ahora bien, este análisis, válido en general para ambas versiones del poema, ha sido hecho pensando sobre todo en la primera pues la segunda versión, al proponer una imagen visual diferente debido a modificaciones de la versificación, y al crear, por eso mismo, modificaciones -visuales y también auditivas- en el ritmo, no deja de producir transformaciones en el sentido.

Una mirada, incluso no necesariamente atenta, a "la figura que reside" en cada una de las versiones del poema nos mostrará que mientras en la primera predomina la impresión de verticalidad y de relativa ingravidez, en la segunda hay un predominio de la horizontalidad, un acortamiento global de la figura y una tendencia al cierre de los versos. La primera versión tiene dieciocho líneas y la segunda quince, pero esta diferencia parece más notoria porque se han eliminado tres líneas breves con el fin de aumentar la extensión de otras tantas. Así, han desaparecido dos tetrasílabos para agregarse en cada caso a un heptasílabo precedente y construir con ello un endecasílabo más firme; también, hacia el final, ha desaparecido un pentasílabo para agregarse al heptasílabo precedente y dar como consecuencia un verso que podría leerse -forzando el sentido- como otro endecasílabo o, respetándolo, como un dodecasílabo. Considerando la segunda versión desde el punto de vista de la organización rítmica, se percibe un debilitamiento estructural. Por ejemplo, al desaparecer el segundo pentasílabo desaparece un metro que tenía la función de apoyo y de eco: ambos pentasílabos (metro en cierto sentido extraño a esta composición pues proviene de la poesía de tradición oral) tenían sin embargo una arquitectura gramatical semejante y una idéntica distribución de los acentos ("está el periódico", "están subiendo"), lo cual servía al mismo tiempo como intervalo y sostén en la marcha prosódica. Por otra parte, entre el primero y el segundo, desde el punto de vis- 
ta temático, hay el paso que va de un anuncio global a una concreción particular de dicho anuncio. Al anularse, pues, este juego de reenvíos y correspondencias, hay, de acuerdo a mi percepción, una considerable disminución del efecto de sentido que el poema procura. En lo que hace a la reducción de los tetrasílabos, ello, creo, reduce la movilidad en la formación de los metros y, por lo tanto, también esa atmósfera de transformaciones tan característica de la primera versión. En la segunda versión no sólo la interacción de los metros parece sufrir una suerte de conflicto sino también el tránsito de lo objetivo a lo subjetivo y, en consecuencia, la función de la voz: por ejemplo, después de una pregunta ("¿Qué será de nosotros...", etc.) que transcurre en una interioridad pues se dirige a un cuerpo dormido, aparece una expresión que debemos atribuir a una voz que irrumpe gradualmente desde el exterior: "Se escucha la respuesta”. De ese modo, el verso que viene -o que venía en la primera versión- indica la conciencia de esa irrupción que ya no es irrupción de la voz sino acercamiento de la violencia del afuera: "Están subiendo". ¿Quién dice "están subiendo"? ¿Hay aquí alguien que habla o este verso nos pone ante una pura escucha? Esa vacilación, que enriquece el sentido, se hace más evidente -se suelta- en la primera versión. La segunda, en cambio, al dejar estos dos momentos de la voz en una sola línea, no da suficiente lugar a ese soltarse. A ello se le agrega la aludida indecisión métrica que hace que esta línea pueda leerse como un endecasílabo (a costa de ignorar la presencia de los dos puntos) o, si se los tiene en cuenta, deba leerse como un dodecasílabo; pero en este último caso estaríamos ante un metro que, pensado desde la organización del sonido que el poema elabora, resulta un metro anómalo. Tal vez todo esto que digo pueda verse globalmente al revisar la figura de la segunda versión en donde se acumulan -hacia el final, en el paso de lo descriptivo a lo narrativo- los metros más extensos y se repite el uso de 
los dos puntos, demasiado visibles para cumplir una función cuyo sentido poético no es demasiado claro.

\section{Final}

Antes de terminar, una advertencia. En la edición de Tarde o temprano que el Fondo de Cultura Económica entregó en 2009, el poema, como era previsible, está otra vez transformado. Esa versión es la que sigue:

\section{Digamos QUe Amsterdam 1943}

El agua vuelve al agua.

Qué inclemente

caer del agua sobre los canales.

Y a lo lejos

un silbato de fábrica.

Entre sábanas, roto, envejeciendo,

está el periódico:

la guerra continúa, la violencia

incendia nuestros ańos.

Bajo tu cuerpo y en tu sueño duermes.

¿Qué será de nosotros? ¿Cuándo y dónde

segará nuestro amor el tajo, el fuego?

Se escucha la respuesta:

Ya llegaron.

Me voy no te despiertes:

los verdugos

han tocado a la puerta.

Ahora estamos ante una composición de 17 líneas. La mano de Pacheco borró la línea que decía "en la mañana inerme", y corrigió la línea en que leíamos "están subiendo" para que ahora leamos: "ya llegaron". Primero hizo desaparecer un dato temporal y afecti- 
vo que nos parecía importante y luego pasó de mostrar una acción en su aspecto durativo ("están subiendo") a mostrarla en su aspecto terminativo, como amenaza consumada: "Ya llegaron". Un cambio es directamente una exclusión y el otro una transformación semántica. Pero, como se puede ver, ninguno de estos dos cambios caen en el campo de observación que hemos escogido para este trabajo. Así, el estudio comparativo de esta última versión podría ser objeto o tema de otro artículo.

\section{Bibliografía}

Cárdenas, Viviana, 2001, "Lingüística y escritura: la zona visuográfica", Tópicos del seminario. Revista de semiótica, núm. 6, "La dimensión plástica de la escritura”, Raúl Dorra (ed.), Puebla, julio-diciembre, pp. 93-141.

Dorra, Raúl, 1986, La literatura puesta en juego, México, UNAM. Jiménez, Juan Ramón, 2008, Obras. Piedra y cielo (1917-1918), José Ramón González (ed.), Miguel Casado (prol.), Madrid, Visor Libros.

Jitrik, Noé, 2001, "La figura que reside en el poema", Tópicos del seminario. Revista de semiótica, núm. 6, "La dimensión plástica de la escritura", Raúl Dorra (ed.), Puebla, julio-diciembre, pp. 13-33.

Lespada, Gustavo, 2002, Esa promiscua escritura, Córdoba, Alción Editora.

Pacheco, José Emilio, 1967, Morirás lejos, México, Joaquín Mortiz. , 1969, No me preguntes cómo pasa el tiempo, México, Joaquín Mortiz.

, 1977, Morirás lejos, 2a ed. revisada, México, Joaquín Mortiz. 
, 1980, Tarde o temprano, México, FCE.

,2009, Tarde o temprano, $4^{\mathrm{a}}$ ed. revisada y aumentada, Ana Clavel (ed.), México, FCE.

Tomás Navarro, Tomás, 1974, Métrica española, Barcelona, Guadarrama. 
